\title{
Analyse du déterminisme de la coloration et de l'ornementation chez la palourde japonaise Ruditapes philippinarum
}

\author{
Jean-Marie Peignon (2), André Gérard ${ }^{(1)}$, Yamama Naciri (1), \\ Christophe Ledu ${ }^{(1)}$ et Pascal Phélipot ${ }^{(1)}$ \\ (1) Adresse pour toute correspondance: IFREMER, Unité de recherche en génétique (URGE), \\ BP 133, Ronce-les-Bains, 17390 La Tremblade, France. \\ (2) IFREMER, Centre de Tahiti, BP 601, Papeete, Tahiti, Polynésie Française.
}

Reçu le 26 décembre 1994; accepté le 22 mars 1995.

Peignon J. M., A. Gérard, Y. Naciri, C. Ledu, P. Phélipot. Aquat. Living Resour., 1995, 8, 181-189.

Analysis of shell colour determinism in the Manila clam Ruditapes philippinarum.

Abstract

Résumé
The Manila clam presents a large polymorphism for shell colours and patterns. No experiment has been conducted to study the determinism of such a polymorphism which has been well known for a long time. The aim of this study was firstly to determine the genetic variation for shell colour and secondly to produce some homozygous lines for the shell colouring. These lines could then be used as controls for laboratory experiments. The colouring is defined by three characters: asymmetry, ornamentation and hue. Clams with asymmetrical colouring are not fully pigmented, except for a posterior band on the left valve. Clams with symmetrical colouring are fully pigmented on the two valves. Four cross series were conducted between the different characters: Asymmetry versus Symmetry, and the Wave [V] and Zebra [Z] ornamentations. These results suggest that colouration is controlled by at least two genes. Asymmetry is controlled by one gene with two alleles; asymmetry (A) is dominant over symmetry (S). Asymmetrical clams are either homozygous or heterozygous and symmetrical ones are therefore always homozygous. Some asymmetrical homozygous clams were identified. A variegated symmetrical phenotype named $\mathrm{Bi}$ appeared in the offspring of the $\mathrm{V} \times \mathrm{V}$ and $\mathrm{Z} \times \mathrm{Z}$ crosses. These results suggest that $\mathrm{V}$ and $\mathrm{Z}$ are dominant over $\mathrm{Bi}$. Nevertheless, other ornamentations (I phenotype for instance) also exist and the gene is probably multiallelic. Determinism of the hue is not defined. Results also show that colouring and sex are not linked. In nearly all the experiments, the observed proportions for the asymmetry are in departure from what was expected under Mendel's hypothesis. This difference is due to a chronic shortage in symmetrical homozygote clams which is still in unexplained.

Keywords: mollusc, bivalve, Manila clam, Ruditapes philippinarum, genetics, Mendelian inheritance, colouration, asymmetry.

La palourde japonaise présente un grand polymorphisme de coloration de la coquille. Bien que ce polymorphisme soit connu depuis longtemps, son hérédité n'a jamais été analysée. Cette étude a pour but, hormis la connaissance fondamentale du déterminisme génétique des caractères de la coloration, de permettre de produire des individus homozygotes pour la coloration. Ceux-ci pourront être utilisés comme marqueurs génétiques dans des expériences au laboratoire. La coloration est définie par trois caractères, l'asymétrie, l'ornementation et la couleur de fond. Les palourdes à coloration asymétrique sont totalement ou partiellement dépigmentées, à l'exception d'une bande postérieure sur la valve gauche. Les palourdes à coloration symétrique sont pigmentées sur les deux valves. Quatre séries de croisements ont permis l'analyse de différents caractères, Asymétrie et Symétrie, et des ornementations Vague [V] et Zébré [Z]. Les résultats suggèrent que la coloration est contrôlée par au moins deux gènes. Le caractère d'asymétrie 
est contrôlé par un gène à deux allèles, asymétrie (A) dominante et symétrie (S) récessive. Quelques individus asymétriques homozygotes ont été identifiés. Un phénotype Bigarré (Bi), est apparu dans les ségrégations du croisement $\mathrm{V} \times \mathrm{V}$ et des croisements $\mathrm{Z} \times \mathrm{Z}$. Les résultats suggèrent que Vague et Zébré sont dominants sur Bigarré. Cependant, il existe d'autres ornementations (phénotype I) et le gène codant pour l'ornementation serait ainsi multiallélique. Le déterminisme de la couleur n'est pas défini. Par contre, les observations montrent que la coloration n'est pas liée au sexe. Dans la majorité des croisements, les proportions observées pour l'asymétrie s'éloignent des rapports attendus selon les lois de Mendel. Cette différence est due à un déficit systématique en individus homozygotes symétriques qui n'a pu être expliqué.

Mots-clés : mollusque, bivalve, palourde, Ruditapes philippinarum, génétique, hérédité mendélienne, coloration, asymétrie.

\section{INTRODUCTION}

Chez les mollusques, le déterminisme de la coloration peut avoir deux origines, environnementale ou génétique. Underwood et Creese (1976), sur Austrocochlea constricta, montrent qu'il existe une relation entre l'aspect extérieur de la coquille et la disponibilité en nourriture. De même chez l'ormeau Haliotis rufescens, un régime à base d'algues rouges entraîne une coloration rouge de la coquille (Leighton, 1961). Par contre, de nombreux auteurs s'accordent sur l'origine génétique du polymorphisme de couleur et d'ornementation, c'est le cas de l'escargot terrestre Cepaea nemoralis (Clarke, 1975; Muray et Clarke, 1989), du bigorneau perceur Urosalpinx cinerea (Cole, 1975) et de la moule Mytilus edulis (Innes et Haley, 1977; Newkirk, 1980). La coloration et l'ornementation de ces espèces sont deux caractères génétiquement indépendants, à ségrégation mendélienne simple.

La palourde japonaise Ruditapes philippinarum (Adams et Reeve) présente un grand polymorphisme de la coloration extérieure de la coquille. Bien que ce polymorphisme soit connu depuis longtemps, aucune étude ayant pour objet son déterminisme n'a été effectuée. Les colorations variées de la palourde ont été bien décrites par Taki (1941) qui les a classées en 4 grands types, «Banded » avec ou sans bande radiale et entièrement pigmentée, «Marked » avec des taches pie ou bigarrées, "Wave " avec des fines rayures brunes disposées en vagues concentriques à partir de l'umbo, «Albino » ̀̀ coquille blanche ou faiblement ornée et colorée, à l'exception de la bande postérieure normalement pigmentée sur la valve gauche. Taki a établi que les différentes ornementations des colorations symétriques sont les mêmes que celles portées par la bande postérieure de la valve gauche de l'Albino ( $f$ ig. 1). En conclusion de son article, Taki a émis, sans la vérifier, l'hypothèse de la transmission selon les lois mendéliennes des caractères de coloration chez Ruditapes philippinarum. Depuis, les seules études récentes sur l'aspect extérieur de la coquille de Ruditapes philippinarum concernent la distribution des stries (Gérard, 1978a) et la formation des stries liées au rythme des marées (Richardson, 1987, 1988).
Hormis la connaissance fondamentale que l'on peut en espérer, l'étude du déterminisme de la coloration et de l'ornementation peut trouver plusieurs applications. Au laboratoire, les caractères de coloration pourraient servir de marqueurs, plus particulièrement en gynogenèse où le sperme irradié de mâle à coloration dominante serait utilisé comme inducteur de fécondation. L'absence de descendants à coloration dominante permettrait d'apprécier le succès de l'expérience. Dans le domaine courant, la coloration peut avoir certaines retombées économiques immédiates. La couleur peut être un critère de choix du consommateur. Ainsi la palourde blanche est appréciée en Asie, alors que la palourde noire est préférée en France, en Italie et en Galice.

L'objectif de cette étude est donc de vérifier la nature génétique de ce déterminisme et de décrire les lois qui le régissent.

\section{MATÉRIEL ET MÉTHODES}

L'observation des palourdes montre que la coloration peut être décrite par trois éléments distincts complémentaires, l'asymétrie, l'ornementation et la couleur ( $f g .1$ ).

Les palourdes asymétriques (phénotype A) présentent une valve gauche à bande postérieure ornée et colorée normalement alors que sur le reste de la coquille, ornementation et coloration ont complètement ou partiellement disparu. Pour les palourdes symétriques (S), les deux valves ont le même aspect par rapport à l'axe matérialisé par la charnière. L'ornementation caractérise les éléments décoratifs qui agrémentent la coquille; elle se traduit par la présence de vagues (dentelures concentriques en forme de triangle à partir de l'umbo : phénotype V), de zébrures (stries concentriques à peu près équidistantes et foncées sur un fond plus pâle : phénotype $Z$ ), de bigarrures (taches sans arrangement précis: phénotype $\mathrm{Bi}$ ), de bandes radiales ou de marbrures pie. L'absence d'ornementation est notée I. Ces éléments sont visibles sur toute la coquille (palourdes symétriques) ou uniquement sur la bande postérieure (palourdes asymétriques). Des bandes radiales étroites, formant un V blanc ou rouge, peuvent se superposer à une autre ornementation. La 
couleur correspond à la teinte de fond de la coquille. Elle peut être blanche, jaune, noire ou bleue.

La méthode utilisée pour déterminer la nature de la transmission des caractères de coloration est celle préconisée par Cain (1988) pour les coquilles de mollusques.

\section{Reproduction et élevage des palourdes}

Les palourdes adultes proviennent d'un élevage du littoral charentais (côte Atlantique, France) où cette activité s'est développée depuis plusieurs années. Leur origine parentale est indéterminée. Elles sont issues de naissains d'écloseries commerciales qui pratiquent l'intercroisement systématique d'un grand nombre de géniteurs, entraînant une grande hétérogénéité des colorations.

En dehors de la période naturelle de reproduction qui se situe de juin à août, la maturation des palourdes est accélérée à partir du mois de février. À cet effet, les palourdes sont stockées dans des bacs de 40 litres à la densité d'un individu par litre d'eau de mer à $22^{\circ} \mathrm{C}$. L'eau, enrichie en phytoplancton, Skeletonema costatum, Pavlova lutheri, Chaetoceros calcitrans, Isochrysis sp. (clone Tahiti) et Tetraselmis suecica, est renouvelée en continu à un débit de 40 litres par heure. En un mois, les palourdes atteignent la maturité sexuelle.

La ponte est provoquée par choc thermique : l'eau est maintenue à $16^{\circ} \mathrm{C}$ pendant quelques minutes. Quand les palourdes siphonnent de nouveau, la température est augmentée progressivement par circulation continue d'eau à $28^{\circ} \mathrm{C}$. Une suspension de sperme provenant d'un mâle sacrifié peut être injectée dans le siphon inhalant afin d'activer l'expulsion des gamètes. Aux premiers signes de ponte qui apparaissent entre $1 \mathrm{~h}$ et $4 \mathrm{~h}$ après le début de l'induction, les palourdes sont rincées puis isolées. La première émission de gamètes n'est pas conservée pour éviter les fécondations incontrôlées. Cette méthode garantie un bon contrôle des croisements bien que l'éventualité d'une «pollution " ne puisse être totalement écartée, à des niveaux très faibles cependant (de l'ordre de 1\%). Les gamètes récoltés séparément sont répartis dans des béchers selon le plan de croisement prévu, produisant ainsi une série de familles de plein-frères ayant, pour certaines d'entre elles, un parent en commun. La fécondation est réalisée à raison de 10 spermatozoïdes par ovule.

Après une incubation de $24 \mathrm{~h}$ à $25^{\circ} \mathrm{C}$ dans de l'eau de mer filtrée à $3 \mu \mathrm{m}$, les larves sont dénombrées et mesurées, leur densité ajustée à 5 par $\mathrm{ml}$ dans un bac de 30 litres. Pendant l'élevage larvaire, dénombrement, mesure et renouvellement de l'eau de mer sont effectués tous les deux jours. Du phytoplancton est apporté quotidiennement à raison de 20 cellules par microlitre d'eau de mer pour chacune des espèces suivantes : Pavlova lutheri, Chaetoceros calcitrans (forma pumilum) et Isochrysis sp. (clone Tahiti).
La métamorphose, qui intervient environ 10 à 12 jours après la fécondation, est précédée par l'apparition du pied et des ébauches branchiales. Les larves devenues pédivéligères cherchent un support par reptation et produisent du byssus pour s'y fixer. Elles mesurent alors 190 à $200 \mu \mathrm{m}$. Transférées en micronurserie pour se métamorphoser, les jeunes palourdes y séjournent jusqu'à une taille de $10 \mathrm{~mm}$ environ, qui rend possible le contrôle des caractères de coloration. Elles sont alors âgées de 90 à 120 jours.

Les élevages ont été effectués sur une période de deux ans et les difficultés zootechniques du début ont ainsi pu être corrigées dans les élevages suivants. La fécondation et la phase larvaire ont été mieux maîtrisées, permettant de mener à terme la majorité des élevages. De même, des précautions plus importantes, prises à tous les stades, ont permis d'éviter les fécondations incontrôlées et les contaminations d'un lot à l'autre.

\section{Croisements effectués}

Quatre séries de croisements ont été effectués permettant d'analyser respectivemient le caractère d'asymétrie/symétrie et deux caractères d'ornementation, vague et zébré. Dans la première série (1-1 à 1-4), les géniteurs asymétriques sont à bande postérieure foncée et les symétriques foncés unis sans ornementation particulière. Tous les géniteurs de la deuxième série (2-2 à 2-18) proviennent de la descendance du croisement 1-1. Les géniteurs symétriques du troisième essai (3-1 et 3-2) sont vagues, ceux du quatrième essai (4-1 à 4-14) présentent pour partie une omementation zébrée. 500 à 2400 individus ont été échantillonnés et répertoriés selon leur couleur pour chaque croisement. Les phénotypes des géniteurs ou des produits de croisement sont présentés sur la figure 1.

Les croisements serviront également à vérifier si le caractère d'asymétrie est lié au sexe.

Le test $\mathrm{du} \chi^{2}$ a été utilisé pour comparer les répartitions observées à la répartition théorique attendue et pour comparer l'homogénéité de plusieurs répartitions. L'utilisation de la correction de Bonferoni, qui consiste à diviser le seuil de signification (en général 5\%) par le nombre de réplicats, permet d'augmenter la validité des résultats lorsque cela est possible (Holm, 1979).

\section{RÉSULTATS}

\section{Analyse du caractère « Asymétrie/Symétrie »}

Les ségrégations obtenues dans les différentes séries de descendances sont classées en quatre groupes, basés uniquement sur les proportions obtenues pour le caractère asymétrie/symétrie : $75 / 25,50 / 50$, tout asymétrique ou tout symétrique $(t a b, 1)$. Les proportions observées dans les descendances sont 


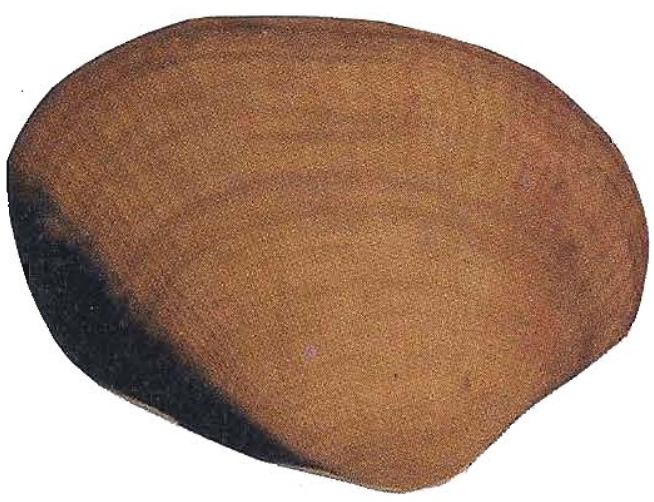

Asymétrique $[\mathrm{A}]$

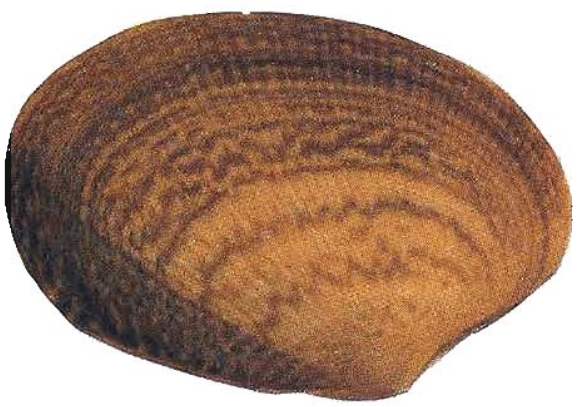

Asymétrique Vague [AV]

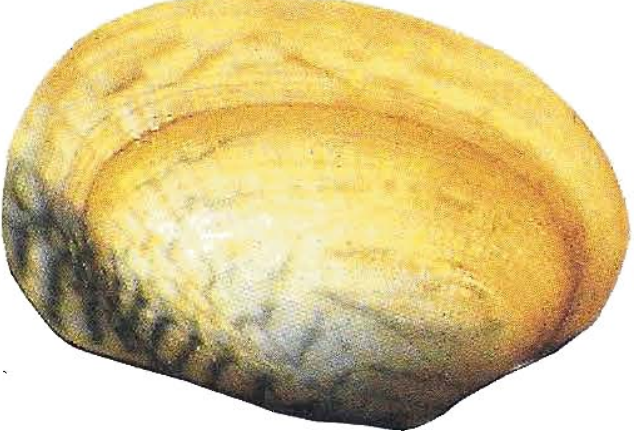

Asymétrique Zébré [AZ]

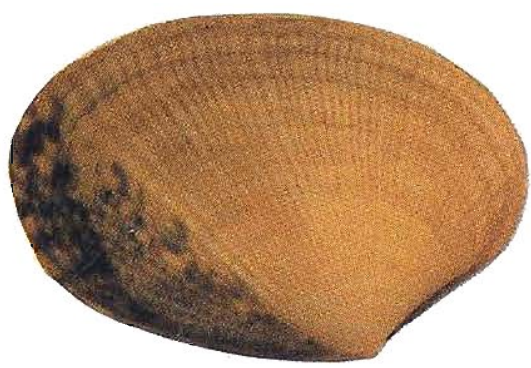

Asymétrique Bigarré [ABi]

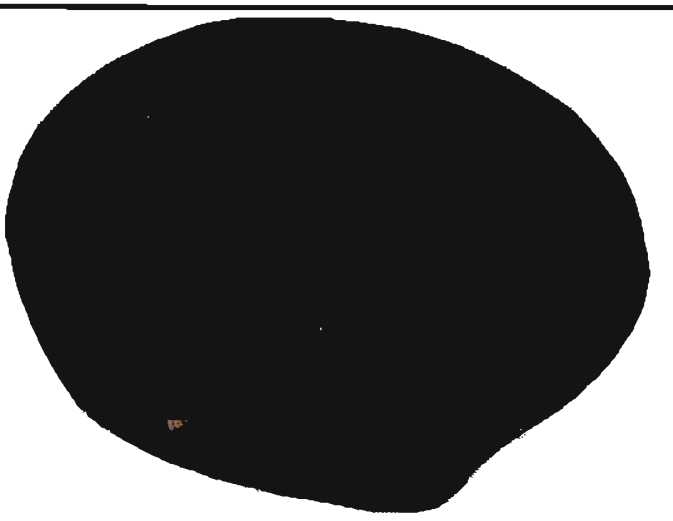

Symétrique [S]

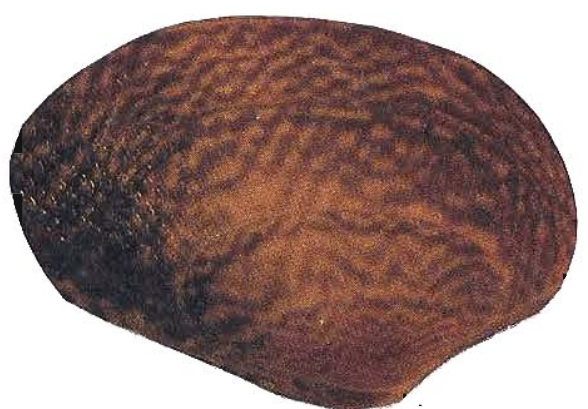

Symétrique Vague [SV]

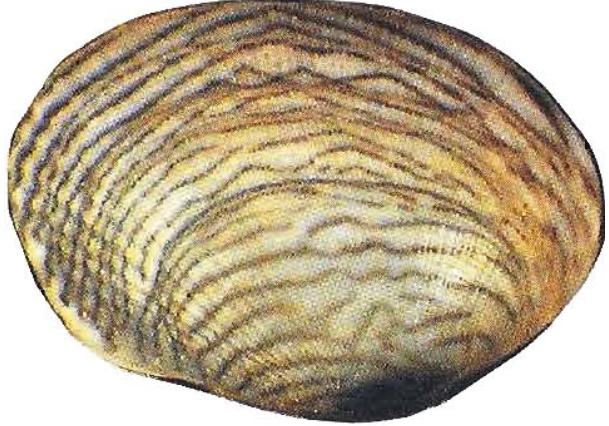

Symétrique Zébré [SZ]

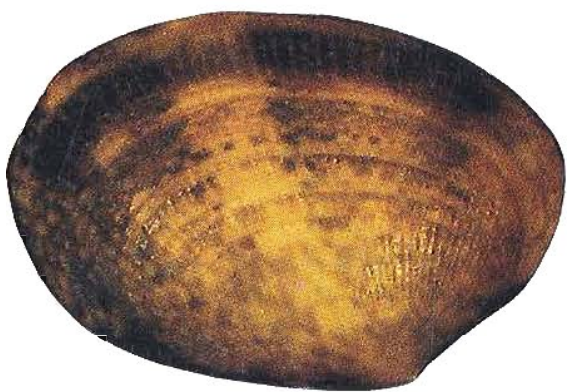

Symétrique Bigarré [SBi]

Figure 1. - Prénulypes el abréviations utilisées.

Phenotypes and abbreviations used. 
Tableau 1. - Résultats de l'analyse du caractère d' "Asymétrie ».

Results on asymmetry character.

\begin{tabular}{|c|c|c|c|c|c|c|}
\hline Lot & 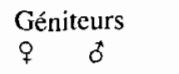 & Origine & {$[\mathrm{A}]$} & {$[\mathrm{S}]$} & Ratio A/S & $x^{2 t}$ \\
\hline $1-1$ & {$[\mathrm{~A}] \times[\mathrm{A}]$} & inconnue & 788 & 226 & $75 / 25$ & $3,98^{\text {nst }}$ \\
\hline $1-3$ & {$[\mathrm{~A}] \times[\mathrm{S}]$} & inconnue & 787 & 658 & $50 / 50$ & $11,51^{* *}$ \\
\hline $1-4$ & {$[\mathrm{~A}] \times|\mathrm{S}|$} & inconnue & 963 & 849 & $50 / 50$ & $7,17^{\mathrm{ns}}$ \\
\hline $2-2$ & {$[\mathrm{~A}]_{1} \times[\mathrm{A}]_{5}$} & $1-1$ & 821 & 176 & $75 / 25$ & $28,5^{* * *}$ \\
\hline $2-6$ & {$[\mathrm{~A}]_{2} \times[S]_{6}$} & $1-1$ & 430 & 432 & $50 / 50$ & $0,002^{\text {ns }}$ \\
\hline $2-7$ & {$[\mathrm{~S}]_{3} \times[\mathrm{A}]_{4}$} & $1-1$ & 1156 & 13 & - & - \\
\hline $2-8$ & {$[\mathrm{~S}]_{3} \times[\mathrm{A}]_{5}$} & $1-1$ & 573 & 556 & $50 / 50$ & $0,26^{\mathrm{ns}}$ \\
\hline $2-10$ & {$[\mathrm{~A}]_{9} \times[\mathrm{A}]_{7}$} & $1-1$ & 2375 & 6 & - & - \\
\hline $2-13$ & $\mid \mathrm{S}]_{10} \times[\mathrm{A}]_{7}$ & $1-1$ & 484 & 54 & - & - \\
\hline $2-14$ & {$[\mathrm{~S}]_{10} \times[\mathrm{S}]_{8}$} & $1-1$ & 11 & 875 & - & - \\
\hline $3-1$ & {$[\mathrm{~S}] \times[\mathrm{A}]$} & inconnue & 320 & 250 & $50 / 50$ & $8,6^{*}$ \\
\hline $3-2$ & {$[S] \times[S]$} & inconnue & - & 950 & - & - \\
\hline $4-1$ & {$[S]_{3^{\prime}} \times[S]_{1}^{\prime}$} & inconnue & - & 983 & - & - \\
\hline $4-2$ & {$[\mathrm{~S}]_{3^{\prime}}^{\prime} \times[\mathrm{S}]_{2^{\prime}}^{\prime}$} & inconnue & - & 1114 & - & - \\
\hline $4-3$ & {$[\mathrm{~S}]_{3^{\prime}}^{\prime} \times[\mathrm{A}]_{12^{\prime}}$} & inconnue & 420 & 480 & $50 / 50$ & $4^{\text {ns }}$ \\
\hline $4-4$ & {$[S]_{3^{\prime}}^{\prime} \times[S]_{13^{\prime}}$} & inconnue & - & 1083 & - & - \\
\hline $4-5$ & {$[S]_{4}^{\prime} \times[S]_{1}^{\prime}$} & inconnue & - & 1280 & - & - \\
\hline $4-6$ & {$[\mathrm{~S}]_{4}^{\prime} \times[S]_{2}^{\prime}$} & inconnue & - & 870 & - & - \\
\hline 4-7 & {$[S]_{4^{\prime}}^{4} \times[\mathrm{A}]_{12^{\prime}}$} & inconnue & 445 & 365 & $50 / 50$ & $7,9^{\mathrm{ns}}$ \\
\hline $4-8$ & {$[S]_{4^{\prime}}^{4} \times[S]_{13^{\prime}}$} & inconnue & - & 920 & - & - \\
\hline $4-9$ & {$[\mathrm{~A}]_{14^{\prime}} \times[\mathrm{S}]_{1^{\prime}}^{\prime}$} & inconnue & 574 & 507 & $50 / 50$ & $4,15^{\mathrm{ns}}$ \\
\hline $4-10$ & {$[\mathrm{~A}]_{14^{\prime}}^{14} \times[\mathrm{S}]_{2^{\prime}}^{\prime}$} & inconnue & 611 & 601 & $50 / 50$ & $0,08^{\mathrm{ns}}$ \\
\hline $4-13$ & {$[S]_{15^{\prime}}^{14} \times[S]_{1^{\prime}}^{2}$} & inconnue & - & 1103 & - & - \\
\hline
\end{tabular}

$\dagger$ Niveau de signification tenant compte de la correction de Bonferroni.

$\dagger \dagger$ Les seuils de signification sont notés ns pour $\alpha>5 \%$, * pour $\alpha \leq 5 \%$, ** pour $\alpha \leq 1 \%$ et *** pour $\alpha \leq 0,1 \%$.

proches des pourcentages théoriques attendus dans le cas d'un caractère monogénique à deux allèles. Pour les descendances en ségrégation (15 croisements sur 32), les tests montrent que les résultats ne sont pas significativement différents des résultats théoriques attendus dans 11 cas sur 15 . Certaines descendances présentent un phénotype unique $(3-1,4-1,4-2,4-4$, 4-5, 4-6, 4-8, 4-13 et 4-14). On considérera également comme homogènes les descendances ayant une très forte proportion d'un des deux phénotypes $(2-4,2-7$, $2-10,2-13,2-14,2-15,2-16$ et $2-18$ ).

L'observation de croisements entre asymétriques [A] montre que les descendances ségrègent dans des proportions proches de 75/25 asymétrique/symétrique (1-1, 1-2, 2-2 et 2-5). Pour ce type de croisement, trois résultats sur quatre sont non significativement différents des résultats théoriques attendus. L'observation de croisements entre asymétriques [A] et symétriques [S] montre que les descendances ségrègent dans des proportions proches de 50/50 asymétrique/symétrique (1-3, 1-4, 2-3, 2-6, 2-8, 2-17, $3-2,4-3,4-7,4-9$ et 4-10). Sept des onze tests sont non significativement différents des résultats théoriques attendus. Des croisements entre asymétriques [A] (2-4, $2-10,2-16$ et 2-18) ainsi que certains croisements entre asymétriques [A] et symétriques [S] (2-7, 2-13 et 2-15) ont donné des descendances entièrement asymétriques. Les croisements entre symétriques [S] ont donné des descendances entièrement symétriques (2-14, 3-1, 4-1, 4-2, 4-4, 4-5, 4-6, 4-8, 4-13 et 4-14). Les descendances entièrement asymétriques ou symétriques sont cohérentes. La présence d'individus symétriques dans des ségrégations théoriques à $100 \%$ asymétriques (et vice-versa) est probablement accidentelle. Elle serait due soit à une faible proportion de fécondations incontrôlées résultant de la méthode d'induction de la ponte, soit à des contaminations entre les lots au cours de l'élevage.

Bien que parfois significativement différents des proportions attendues, ces résultats permettent cependant de fonder l'hypothèse que l'asymétrie est contrôlée par un gène à deux allèles, asymétrie dominant et symétrie récessif. Selon la même hypothèse, les phénotypes asymétriques $[\mathrm{A}]$ issus des 
croisements entre hétérozygotes AS, sont pour $1 / 3$ homozygotes AA et $2 / 3$ hétérozygotes AS.

Le génotype de quelques-uns des reproducteurs utilisés peut être déduit des résultats précédents. Certains géniteurs asymétriques du deuxième essai, en donnant une descendance entièrement asymétrique qu'ils soient croisés avec un asymétrique ou un symétrique, se sont révélés homozygotes AA pour ce caractère. C'est le cas des mâles A4, A7 et A11. Les autres asymétriques sont hétérozygotes AS, c'est le cas du mâle A5 et des femelles A1, A2, A 12, A 12' et $\mathrm{A} 14^{\prime}$. Tous les géniteurs de phénotype symétrique sont homozygotes SS. Le génotype de la femelle A9 ne peut être déterminé, le seul croisement viable avec le mâle A7 homozygote est en effet insuffisant pour conclure.

\section{Analyse des caractères d'ornementation «Vague » et «Zébré »}

Le croisement 3-1 d'une femelle symétrique vague [SV] et d'un mâle asymétrique d'ornementation non déterminée $[\mathrm{A}]$ a donné 570 individus ségrégeant dans des proportions proches de $25 \%$ des phénotypes suivants : $28,2 \%$ d'asymétriques à bande postérieure vague $[\mathrm{AV}], 27,9 \%$ d'asymétriques à bande postérieure bigarrée [ABi], 24,2\% de symétriques vagues [SV] et 19,6\% de symétriques bigarrés [SBi]. Deux hypothèses peuvent être avancées : la première consiste à supposer que l'ornementation du mâle asymétrique était de type bigarrée mais qu'elle n'a pas été retenue comme telle au moment du croisement. Cette omission s'expliquerait par le fait que la description des ornementations s'est affinée au fur et à mesure de l'obtention des descendances et que souvent les parents n'existaient plus à ce moment-là. Dans ce cas, et en supposant l'existence d'un troisième allèle supplémentaire I, correspondant à l'absence d'ornementation, tel que $\mathrm{V}>\mathrm{Bi}>\mathrm{I}$, la femelle serait de génotype SSVBi et le mâle de génotype ASBiBi ou ASBiI. La seconde hypothèse suppose comme précédemment que 3 allèles différents ségrègent au même locus d'ornementation ( $\mathrm{V}>\mathrm{Bi}>\mathrm{I})$ mais que le mâle est de phénotype [AI]. Les parents seraient alors de génotype SSVBi et ASII. Que ce soit dans un cas ou dans l'autre, l'analyse statistique des résultats donne un test non significativement différent des résultats théoriques attendus pour des rapports $\mathrm{V} / \mathrm{Bi}$ de $50 / 50$, et suggère que Vague, Bigarré et l'éventuel allèle supplémentaire I sont les allèles d'un même gène codant pour l'ornementation.

Le croisement 3-2 entre deux palourdes de phénotype symétrique vague [SV] a donné dans sa descendance $66,3 \%$ d'individus de phénotype parental vague [SV], et deux phénotypes non-parentaux $31,4 \%$ d'individus Bigarrés [SBi] et 2,3\% d'individus à coquille lisse et brune (tab. 2). Tous ces individus sont symétriques comme prévu. L'hypothèse testée, celle d'un gène d'ornementation à 2 allèles, l'un récessif car n'apparaissant que dans la descendance
Tableau 2. - Résultats de l'analyse des caractères d'ornementation «Vague " et "Zébré ".

Results of "wave" and "zehra" omamentation analyses.

\begin{tabular}{|c|c|c|c|c|c|}
\hline Vague & $q^{\text {Géniteurs }}$ & {$[V]$} & [Bi] & $\begin{array}{l}\text { Ratio } \\
\text { V/Bi }\end{array}$ & $\chi^{2}$ \\
\hline $\begin{array}{l}3-1 \\
3-2\end{array}$ & $\begin{array}{l}{[\mathrm{SV}] \times[\mathrm{AI}]} \\
{[\mathrm{SV}] \times[\mathrm{SV}]}\end{array}$ & $\begin{array}{l}300 \\
652\end{array}$ & $\begin{array}{l}270 \\
298\end{array}$ & $\begin{array}{l}50 / 50 \\
75 / 25\end{array}$ & $\begin{array}{c}1,38^{n 4} \\
20,55^{* * *}\end{array}$ \\
\hline Zébré & $q_{q}^{\text {Géniteurs }}$ & {$[Z]$} & {$[\mathrm{Bi} \mid$} & $\begin{array}{c}\text { Ratio } \\
\text { Z/Bi }\end{array}$ & $\chi^{2+}$ \\
\hline $\begin{array}{l}4-1 \\
4-2 \\
4-3 \\
4-4 \\
4-5 \\
4-6 \\
4-7 \\
4-8 \\
4-9 \\
4-10 \\
4-13 \\
4-14\end{array}$ & $\begin{array}{l}{[\mathrm{SZ}]_{3} \times[\mathrm{SZ}]_{1}} \\
{[\mathrm{SZ}]_{3} \times[\mathrm{SZ}]_{2}} \\
{[\mathrm{SZ}]_{3} \times[\mathrm{AI}]_{12}} \\
{[\mathrm{SZ}]_{3} \times[\mathrm{SI}]_{13}} \\
{[\mathrm{SZ}]_{4} \times[\mathrm{SZ}]_{1}} \\
{[\mathrm{SZ}]_{4} \times[\mathrm{SZ}]_{2}} \\
{[\mathrm{SZ}]_{4} \times[\mathrm{AI}]_{12}} \\
{[\mathrm{SZ}]_{4} \times[\mathrm{SI}]_{13}} \\
{[\mathrm{AI}]_{14} \times[\mathrm{SZ}]_{1}} \\
{[\mathrm{AI}]_{14} \times[\mathrm{SZ}]_{2}} \\
{[\mathrm{SI}]_{15} \times[\mathrm{SZ}]_{1}} \\
{[\mathrm{SI}]_{15} \times[\mathrm{SZ}]_{2}}\end{array}$ & $\begin{array}{l}713 \\
814 \\
474 \\
545 \\
920 \\
646 \\
407 \\
432 \\
559 \\
622 \\
591 \\
289\end{array}$ & $\begin{array}{l}270 \\
300 \\
426 \\
538 \\
360 \\
224 \\
403 \\
488 \\
522 \\
590 \\
512 \\
241\end{array}$ & $\begin{array}{l}75 / 25 \\
75 / 25 \\
50 / 50 \\
50 / 50 \\
75 / 25 \\
75 / 25 \\
50 / 50 \\
50 / 50 \\
50 / 50 \\
50 / 50 \\
50 / 50 \\
50 / 50\end{array}$ & $\begin{array}{l}3,19^{\mathrm{nst}} \\
2,21^{\mathrm{ns}} \\
2,56^{\mathrm{ns}} \\
0,05^{\mathrm{ns}} \\
6,67^{*} \\
0,26^{\mathrm{ns}} \\
0,02^{\mathrm{ns}} \\
3,41^{\mathrm{ns}} \\
1,27^{\mathrm{ns}} \\
0,85^{\mathrm{ns}} \\
5,66^{\mathrm{ns}} \\
4,35^{\mathrm{ns}}\end{array}$ \\
\hline
\end{tabular}

+ Niveau de signification tenant compte de la correction de Bonferroni.

$\dagger+$ Les seuils de signification sont notés ns pour $\alpha>5 \%$, * pour $\alpha \leq 5 \%$ et $* * *$ pour $\alpha \leq 0,1 \%$.

(Bi) et l'autre dominant (V), et de ce fait celle d'une ségrégation $75 / 25$, n'est pas validée par les résultats. La présence d'individus atypiques en faible proportion est vraisemblablement due à des contaminations, comme c'est déjà le cas dans les deux premières séries de croisements. L'hypothèse de la présence de 3 allèles $\mathrm{Z}>\mathrm{Bi}>\mathrm{I}$, l'un des parents étant de génotype $\mathrm{ZBi}$ et l'autre de génotype ZI ne modifie pas l'interprétation, la ségrégation attendue étant toujours la même.

L'hypothèse de l'existence de 3 allèles $(\mathrm{Z}>\mathrm{Bi}>\mathrm{I})$ permet également l'interprétation de la $4^{\mathrm{e}}$ série de croisements faisant intervenir une nouvelle ornementation zébrée (Z). Seuls les parents de 4 croisements (4-1, 4-2, 4-5 et 4-6) ont été clairement décrits. Il s'agit d'individus symétriques zébrés donnant des ségrégations $\mathrm{Z} / \mathrm{Bi}$ de $75 / 25$. Trois des quatre tests valident l'hypothèse de la présence de 2 allèles $\mathrm{Z}$ et $\mathrm{Bi}$, avec $\mathrm{Z}>\mathrm{Bi}$, les parents étant alors hétérozygotes $\mathrm{ZBi}$ pour le gène d'ornementation. Les croisements 4-3, 4-4, 4-7 à 4-14 ont été effectués entre les mêmes palourdes symétriques zébrées d'une part et des palourdes à ornementation indéfinie (I) symétriques ou asymétriques d'autre part. Dans tous les cas, les ségrégations obtenues ne sont pas significativement différentes des proportions $50 / 50$ en $\mathrm{Z} / \mathrm{Bi}$. En conservant l'hypothèse que $\mathrm{Z}>\mathrm{Bi}>\mathrm{I}$, les phénotypes $[\mathrm{AI}]$ ou $[\mathrm{SI}]$ sont forcément homozygotes pour l'allèle I. De ce fait, les individus $[\mathrm{SZ}]_{1},[\mathrm{SZ}]_{2}$. $[S Z]_{3}$ et $[S Z]_{4}$ sont hétérozygotes $\mathrm{ZBi}$ pour le gène d'ornementation. 
Parmi les géniteurs, aucun génotype homozygote pour le caractère Vague ou Zébré n'a pu être mis en évidence.

\section{Ornementations supplémentaires}

Deux croisements (4-4 et 4-8) issus d'un même mâle symétrique, ont présenté une modification de l'expression des ornementations zébrée et bigarrée, celles-ci étant pour moitié soit jaune, soit foncée. De la même façon, toute la descendance bigarrée d'un des mâles symétriques zébrés présentait une modification de l'ornementation par juxtaposition d'une double bande blanche symétrique disposée en $\mathrm{V}$. Par ailleurs, deux individus asymétriques particuliers ont été observés dans les descendances étudiées. L'un avec une bande supplémentaire en position antérieure sur la valve gauche et l'autre avec la valve droite à ornementation vague normale, alors que l'ornementation aurait dû être très atténuée. Leur faible fréquence d'apparition (deux individus sur quelques dizaines de milliers) laisse à penser qu'il s'agit d'une mutation spontanée.

\section{Indépendance des caractères d'asymétrie et d'ornementation}

Les résultats présentés ne permettent pas de statuer sur l'indépendance des gènes d'asymétrie et d'ornementation. En effet, que ce soit dans la série de croisements faisant intervenir l'ornementation Vague ou dans celle faisant intervenir l'ornementation Zébrée, nul croisement entre doubles hétérozygotes n'est disponible (AS-ZBi ou AS-VBi).

\section{Le caractère d'asymétrie est-il lié au sexe?}

La palourde ne présente pas de caractères sexuels secondaires permettant de déterminer le sexe d'un individu par son aspect extérieur. Elle n'a par ailleurs pas d'hétérochromosomes (Gérard, 1978b).

La liaison de la coloration au sexe a été vérifiée en testant les ségrégations 50/50 dans les croisements réciproques entre asymétriques et symétriques des différents essais (tabl. 1). Le test d'homogénéité effectué, portant sur les descendances des croisements q $[\mathrm{A}] \times \delta[\mathrm{S}](2252[\mathrm{~A}]$ et 2079 [S]) et sur les croisements $q[\mathrm{~S}] \times \delta[\mathrm{A}](1758[\mathrm{~A}]$ et 1651 [S]), montre que les résultats ne dépendent pas du sens du croisement (d.d.l. $=1, \chi^{2}=0,14, \alpha>0,90$ ). Le gène d'asymétrie ne montre donc pas de liaison au sexe.

\section{Déficit en homozygotes symétriques}

Un déficit en phénotypes symétriques homozygotes récessifs a été observé dans 12 croisements sur 14 , quel que soit le type de ségrégation et d'ornementation associé (tabl. 1). Les pourcentages observés dans les ségrégations $75 / 25$ ont montré un déficit moyen de $1.9 \%$ en homozygotes symétriques (calculé sur l'ensemble des descendances) et ces résultats sont homogènes d'un croisement à l'autre (d.d.l. =2, $\chi^{2}=2,252, \alpha>0,30$, tabl. 3). Un déficit moyen de $3.1 \%$ en homozygotes symétriques récessifs [S] est également constant dans les ségrégations $50 / 50$ (tabl. 3). Les résultats sont homogènes (d.d.l. $=9$, $\left.\Sigma \chi^{2}=16,44, \alpha>0,05\right)$ lorsqu'est éliminé le croisement 4-3 qui montre une tendance inverse (3.3\% d'excès en homozygotes symétriques).

Tableau 3. - Étude de l'homogénéité du déficit en pourcentage (\%) d'homozygotes symétriques (SS) dans les ségrégations $75 / 25$ et 50/50 et tests $\chi^{2}$ sur les tableaux de contingence.

Consistency of symmetric homozygous deficiencies (SS) in 75/25 et 50/50 ratios and $\chi^{2}$ tests on contingency tables.

\begin{tabular}{|c|c|c|c|c|c|}
\hline Croisements & $\begin{array}{l}\text { Géniteurs } \\
q \quad \delta\end{array}$ & $\begin{array}{l}{[\mathrm{A}] /[\mathrm{S}]} \\
\text { observé }\end{array}$ & $\begin{array}{l}{[\mathrm{A}] /[\mathrm{S}]} \\
\text { théorique }\end{array}$ & $\begin{array}{c}\text { Déficit en SS } \\
(\%)\end{array}$ & $\chi^{2}$ partiels \\
\hline $\begin{array}{l}1-1 \\
1-2 \\
2-5\end{array}$ & $\begin{array}{l}{[\mathrm{A}] \times[\mathrm{A}]} \\
{[\mathrm{A}] \times[\mathrm{A}]} \\
{[\mathrm{A}] \times[\mathrm{A}]}\end{array}$ & $\begin{array}{c}788 / 226 \\
1699 / 556 \\
815 / 250\end{array}$ & $\begin{array}{c}773 / 241 \\
1718 / 537 \\
811 / 254\end{array}$ & $\begin{array}{l}2,7 \\
0,3 \\
1,5\end{array}$ & $\begin{array}{l}1,30 \\
0,89 \\
0,07\end{array}$ \\
\hline \multicolumn{5}{|c|}{$\chi^{2}(2 \mathrm{ddl})$ sur les ségrégations $75 / 25$} & $2,52^{\mathrm{ns}}$ \\
\hline $\begin{array}{l}1-3 \\
1-4 \\
2-3 \\
2-6 \\
2-8 \\
2-17 \\
3-1 \\
4-7 \\
4-9 \\
4-10\end{array}$ & $\begin{array}{l}{[\mathrm{A}] \times[\mathrm{S}]} \\
{[\mathrm{A}] \times[\mathrm{S}]} \\
{[\mathrm{A}] \times[\mathrm{S}]} \\
{[\mathrm{A}] \times[\mathrm{S}]} \\
{[\mathrm{S}] \times[\mathrm{A}]} \\
{[\mathrm{A}] \times[\mathrm{S}]} \\
{[\mathrm{A}] \times[\mathrm{S}]} \\
{[\mathrm{S}] \times[\mathrm{A}]} \\
{[\mathrm{A}] \times[\mathrm{S}]} \\
{[\mathrm{A}] \times[\mathrm{S}]}\end{array}$ & $\begin{array}{l}787 / 658 \\
963 / 849 \\
637 / 539 \\
430 / 432 \\
573 / 556 \\
652 / 530 \\
320 / 250 \\
445 / 365 \\
574 / 507 \\
611 / 601\end{array}$ & $\begin{array}{l}768 / 677 \\
963 / 849 \\
625 / 551 \\
458 / 404 \\
600 / 529 \\
628 / 554 \\
303 / 267 \\
430 / 380 \\
574 / 507 \\
644 / 568\end{array}$ & $\begin{array}{r}4,5 \\
3,2 \\
4,2 \\
-0,1 \\
0,8 \\
5,2 \\
6,1 \\
4,9 \\
3,1 \\
0,4\end{array}$ & $\begin{array}{l}1,04 \\
0 \\
0,51 \\
3,63 \\
2,55 \\
1,97 \\
2,08 \\
1,07 \\
0 \\
3,58\end{array}$ \\
\hline
\end{tabular}

$\dagger \dagger$ Le seuil de signification est noté ns pour $\alpha>5 \%$.

Aquat. Living Resour., Vol. 8, n 2 - 1995 


\section{DISCUSSION-CONCLUSION}

Si la description de la coloration des valves de la palourde peut se faire avec les trois caractéristiques majeures d'asymétrie, d'ornementation et de couleur, la présente étude n'apporte un début de réponse que pour les deux premiers. Les résultats observés sont proches des résultats théoriques attendus, mais certains d'entre eux en sont significativement différents. Cette tendance s'atténue au fil des essais, puisque c'est le cas de 5 croisements sur 10 dans les essais 1 et 2 et de seulement un croisement sur 12 dans l'essai 4 . Cette évolution met en évidence la meilleure maîtrise des croisements et des élevages qui a été acquise au cours des deux ans d'expérimentation.

L'analyse des descendances montre que le déterminisme génétique du caractère asymétrie correspond à celui d'un gène à 2 allèles, l'Asymétrie (A) étant dominante et la Symétrie (S) étant récessive. Des individus asymétriques homozygotes dominants ont pu être identifiés.

L'ornementation semble être également un caractère simple, contrôlé par un seul gène mais présentant pour sa part plus de deux allèles. L'analyse de deux ornementations, Vague $(\mathrm{V})$ et Zébrée $(\mathrm{Z})$, a révélé l'existence d'une troisième ornementation appelée bigarrée ( $\mathrm{Bi})$, voire d'une quatrième correspondant à l'absence d'ornementation et notée I. On peut émettre l'hypothèse qu'une série allélique est présente au locus d'ornementation, avec les allèles Zébré et Vague dominants sur l'allèle Bigarré, lui-même dominant sur l'allèle I. Les relations de dominance entre Zébré et Vague n'ont pu être déterminées. Contrairement au cas précédent, aucun individu homozygote n'a pu être identifié pour les ornementations Vague et Zébrée. Les individus bigarrés sont homozygotes $\mathrm{BiBi}$ ou hétérozygotes BiI.

Les croisements réciproques entre asymétrique (AS) et symétrique (SS), ont donné des ségrégations équivalentes dans les deux sens pour ces caractères. Ceci démontre que le caractère asymétrie n'est pas lié au sexe.

L'apparition d'ornementations supplémentaires (présence de bandes radiales de différentes couleurs), de modifications de la forme des bandes ainsi que de nouvelles couleurs de fond (jaune, noire) amène à formuler l'hypothèse de l'existence de gènes supplémentaires.

La diversité des colorations observées met en évidence un système complexe présent également chez d'autres mollusques bien étudiés parce que plus facile à élever comme les escargots Cepaea nemoralis Wolda, 1969; Jones et al., 1977; Cain, 1988; Murray, 1989) et Helix aspersa (Albuquerque de Matos, 1989).

\section{Perspectives}

L'utilisation des palourdes mâles de phénotype asymétrique dominant est possible dans les expériences de gynogenèse réalisées avec des femelles de phénotype symétrique récessif. Une inactivation incomplète du sperme ferait apparaître une partie de la descendance de même phénotype que le père. L'analyse du déterminisme des caractères de coloration de la coquille et du manteau de l'huître creuse Crassostrea gigas pourrait présenter le même intérêt. Dans un esprit de diversification conchylicole, la connaissance du déterminisme de la coloration permettrait de produire des palourdes ou des huîtres colorées à la demande du marché comme cela est fait pour le tilapia (McAndrew et al., 1988; Tave et al., 1989).

\section{RÉFÉRENCES}

Albuquerque de Matos R. M. 1989. Contribution à l'étude des relations entre caractères qualitatifs et quantitatifs chez Helix apersa. Haliotis 19, 153-164.

Cain A. J. 1988. The scoring of polymorphic colour and pattern variation and its genetic basis in molluscan shells. Macologia 28, 1-15.

Clarke B. 1975. The causes of biological diversity. Sci. Am. 233, 50-60.

Cole J. T. 1975. Inheritance of juvenile shell colour of the oyster drill Urosalpinx cinerea. Nature 257, 794-795.

Gérard A. $1978 \mathrm{a}$. Recherche sur la variabilité de diverses populations de Ruditapes decussatus et Ruditapes philippinarum (Veneridac, Bivalvia). Thèse dr. $3^{\text {e }}$ cycle. Univ. Bretagne Occidentale, Brest, France, 149 p.

Gérard A. $1978 b$. Étude des garnitures chromosomiques de deux Veneridae: Ruditapes decussatus (L.) et Ruditapes philippinarum (Adams et Reeve). Haliotis 9, 69-71.

Holm S. 1979. A simple sequentially rejective multiple test procedure. Scand. J. Statist. 6, 65-70.

Innes D. J., L. E. Haley 1977. Inheritance of a shell-color polymorphism in the mussel. J. Hered. 68, 203-204.

Jones J. S., B. H. Leith, P. V. Rawlings 1977. Polymorphism in Cepaea: a problem with too many solutions? Ann. Rev. Ecol. Syst. 8, 109-143.

Leighton D. L. 1961. Observations of the effect of diet on shell coloration in the red abalone. Haliotis rufescens Swainson. Veliger 4, 29-32.

McAndrew B. J., F. R. Roubal, R. J. Roberts, A. M. Bullock, I. M. McEwen 1988. The genetics and histology of red, blond and associated colour variants in Oreochromis niloticus. Genetica 76, 127-137.

Murray J., B. Clarke 1989. The pale brown allele in Cepaea nemoralis. Biol. J. Linn. Soc. 36, 49-54.

Newkirk G. F. 1980. Genetics of shell color in Mytilus edulis (L.) and the association of growth rate with shell color. J. Exp. Mar. Biol. Ecol. 47, 89-94.

Richardson C. A. 1987. Tidal bands in the shell of the clam Tapes phippinarum (Adams \& Reeve, 1850). Proc. $R$. Soc. London 230B, 367-387.

Richardson C. A. 1988. Exogenous and endogenous rhythms of band formation in the shell of the clam Tapes philippinarum (Adams \& Reeve, 1850). J. Exp. Mar. Biol. Ecol. 122, 105-126.

Taki I. 1941. On the variation in the colour pattern of a bivalve, Venerupis philippinarum, with special reference to its bilateral asymmetry. Venus 11, 71-87. 
Tave D., M. Rezk, R. O. Smitherman 1989. Genetics of body color on Tilapia mossambica. J. World Aquac. Soc. 20, 214-222.

Underwood A. J., R. G. Creese 1976. Observation on the biology of the trochid gastropod Austrocochlea constricta
(Lamark) (Prosobranchia). II. The effects of available food on shell-banding pattern. J. Exp. Mar. Biol. Ecol. 23, 229-240.

Wolda H. 1969. Genetics of polymorphism in the land snail, Cepaea nemoralis. Genetica $40,475-502$. 